\title{
Redox Signaling Induced Laminin Receptor RPSA Mediates Cell Adhesion Following Radiofrequency Glow Discharge Treatments
}

\author{
Sasikumar Ponnusamy \\ University at Buffalo \\ Hanan H. Ali \\ University at Buffalo \\ Felisha Dutt \\ University at Buffalo \\ Saeed Ur Rahman \\ University at Buffalo \\ Ahmad A. Salah \\ University at Buffalo \\ Mahek Pipalia \\ University at Buffalo \\ Robert E Baier \\ University at Buffalo \\ Praveen R. Arany ( $\nabla$ prarany@buffalo.edu ) \\ University at Buffalo
}

\section{Research Article}

Keywords: Radiofrequency glow discharge, reactive oxygen species, cell adhesion.

Posted Date: January 27th, 2022

DOI: https://doi.org/10.21203/rs.3.rs-1277848/v1

License: (1) (1) This work is licensed under a Creative Commons Attribution 4.0 International License.

Read Full License 


\section{Abstract}

Current biomaterials are effective at replacing biological structures, but are limited by infections and longterm material failures. In this study, we examined the molecular mechanisms of Radio Frequency Glow Discharge Treatments (RFGDT) in mediating disinfection of biomaterial surfaces and concurrently promoting cell attachment and proliferation. Dental biomaterials were subjected to RFGDT and viability of oral microbial species namely Streptococcus mutants (SM), Streptococcus gordonii (SG), Moraxella catarrhalis (MC), and Porphyromonas gingivalis (PG) were assessed. Cell attachment and survival of a pre-odontoblast cell line, MDPC-23, was examined. Finally, mechanistic investigations into redox generation and biological signaling were investigated. Dental biomaterials induced reactive ozygen species (ROS) following dose-dependent RFGDT based on their compositions. RFGDT reduced microbial viability in the catalase-negative (SM and SG) species more effectively than catalase-positive (MC and PG) species. Cell adhesion assays noted improved MDPC-23 attachment and survival. Pretreatments with $\mathrm{N}$-acetylcysteine (NAC) and catalase abrogated these responses. Immunoassays noted redox-induced downstream expression of a laminin receptor, RPSA, following RFGDT. Thus, RFGDT-induced redox mediates antimicrobial and improved cell responses such as adhesion and proliferation. These observations together provide a mechanistic rationale for the clinical utility of RFGDT with dental biomaterials for regenerative clinical applications.

\section{Introduction}

Biomaterials have enabled tissue engineering to evolve from an emerging science to its current pivotal role in spearheading clinical regenerative medicine. ${ }^{1-3}$ These biomaterial systems have been readily adopted in various clinical fields for esthetics and functional replacements. There has been tremendous progress in biomaterial systems from passive, biocompatible carriers or inert replacements to current bioactive or smart systems capable of sense-and-respond capabilities. ${ }^{4,5}$ Advances in these fields have encompassed newer compositions, optimized nanotopological features, biophysically-actuated and controlled-release biomaterial systems, among many others. These advancements are tied intimately with progress in development and stem cell biology, enabling a critical understanding of cell regulation during tissue healing and regeneration. ${ }^{6-8}$ These include intrinsic regulation with key transcriptional factors, gene organization, signaling, and metabolomics, and extrinsic regulation with membrane, nanoscale ligand engagement, and paracrine signaling. Moreover, among these extrinsic factors, the role of metagenomics from the pervasive microbiota is now better understood, further emphasizing the need for effective disinfection. ${ }^{9-13}$

The oral cavity presents several unique challenges with its soft and hard tissue biological constituents. These oral tissues represent an exclusive anatomical niche where hard tissue (teeth) is exposed through soft tissue (gingiva) via a delicate but rigorous interface. The importance of a healthy oral microbiome

and the impact of dysbiosis in oral and systemic health has been well established. ${ }^{14-16}$ These aspects present significant challenges for biomaterial replacements due to the constant biomechanical, 
microbiological assault, and immunological surveillance in the oral environment. Nonetheless, dentistry has very effectively utilized ceramics, metal, polymers, and their combinations as restorations, prostheses, or implants that are integral to current clinical care. Several recent technologies such as digital impressions, high-resolution cone-beam computed tomography (CBCT), subtractive, and additive 3D printing have accelerated sophistication in device design and reduced production timelines enabling chair-side fabrication. ${ }^{17}$ These advances with custom-fabricated biomaterial devices further emphasize the need to explore effective, in-office disinfection techniques.

An ideal approach must provide disinfection immediately prior to clinical use and must not undermine physical or chemical material performance while optimally preparing the biomaterial interface to promote favorable host biological responses. ${ }^{18,19}$ Various disinfection approaches from dry and wet heat, chemical disinfection, and biophysical means such as ultraviolet, ethylene dioxide gas, carbon dioxide, ultrasound, plasma, microwaves have been employed. ${ }^{20,21}$ Due to their device characteristics, several of these effective disinfection sources are restricted to commercial production and impractical for routine clinical use. Generation of plasma by glow discharge results from ionization of gaseous molecules termed the Townsend avalanche or discharge. ${ }^{22,23}$ This process can be facilitated at both atmospheric and low pressures resulting in a non-thermal process that minimally alters target surfaces. Several power sources have been utilized to generate glow-discharge plasma, such as direct current, electron cyclotron resonance, and radiofrequency. Among them, radiofrequency glow discharge treatment (RFGDT) is capable of generating a large volume of uniformly charged plasma that can flow through both conductive (metal) non-conductive (polymers) biomaterials. RFGDT-generated plasma, in the presence of air or oxygen, removes organic contaminants by generating highly reactive oxygen species (ROS) that promote surface oxidation and hydroxylation. ${ }^{24}$ RFGDT has also been noted to create high surface energy that increases hydrophilicity which facilitates cell spreading and attachment. ${ }^{25-27}$ However, the precise mechanism mediating these improved cell adhesion following RFGDT has not been elucidated. This study investigated the effects of RFGDT on different dental biomaterial surfaces. The concurrent effects on disinfection of four common oral microbes and concurrent changes in cell adhesion and proliferation in an oral cell line, MDPC-23, were assessed. Finally, the precise biological mechanism mediating the cellular responses was investigated using biochemical and molecular assays.

\section{Results}

\section{Redox generation at biomaterial interfaces following RFGDT}

The plasma generated by RFGDT affects the surface chemistry of the biomaterials based on their composition and the presence of exogenous gases. As the dental pulp is exposed to various restorative biomaterials such as glass ionomer cement, polymers, and metals, we examined the generation of redox species following RFGDT with chemiluminescence luminol assay. ${ }^{28}$ RFGDT generated a significant increase in ROS from glass (Fig. 1a) and platinum surfaces (Fig. 1b), while minimal expression was noted on a polymeric (Polymethylmethacrylate, PMMA) surface (Fig. 1c). RFGDT induced a dose- 
dependent increase in redox generation with optimal noted at 180 seconds (Fig. 1d). As a prior study utilized various surface treatments such as detergent, alcohol, or silane, we noted only RFGDT generates redox (Fig. 1e). These results show that while multiple agents are capable of disinfecting biomaterial interfaces, induction RFGDT-induced redox could persistently prevent microbial colonization while favorably modulating host cell interactions.

\section{Microbial colonization is inhibited by RFGDT-induced redox}

To examine the disinfection potential of RFGDT, we first examined the endogenous antioxidant by incubating individual microbe with hydrogen peroxide. We noted that Streptococcus gordonni and Streptococcus mutants, which are catalase-negative, failed to generate effervescence (Fig. 2a). In contrast, Moraxella catarrhalis and Porphyromonas gingivalis, which are catalase-positive, generated profuse effervescence indicating the active ROS neutralization capabilities. Glass coverslips subjected to RFGDT were seeded with $S$. mutant and demonstrated reduced viability with the live-dead assay (Fig. 2b). Consistent with this observation, colony-forming units (CFUs) of S. mutants (Fig. 2c) and S. gordonni (Fig. 2d) were significantly reduced following RFGDT. The reduction in microbial survival could be prevented by pre-incubation with ROS scavengers $\mathrm{N}$-acetyl cysteine (NAC) and catalase. Consistent with these observations, no differences in CFUs were observed with M. catarrhalis (Fig. 2e) and $P$. gingivalis (Fig. 2f) seeded on glass coverslips following RFGDT. These results demonstrate RFGDT-induced redox reduces viability and proliferation of susceptible (catalase-negative) microbes, while anaerobic, catalasepositive species were resistant.

\section{RFGDT improves cell adhesion and expansion}

Cell adhesion to the substrate is a crucial parameter for attachment and subsequent proliferation. We next examined the effects of RFGDT on a mammalian cell line, MDPC-23. Glass coverslips were subjected to detergent, alcohol, silane (ODS), or RFGDT prior to seeding. We examined both weak and strong cell adhesion by assessing basal and centrifugal force-induced adherent and floating cell populations at 5 and 24 hours after cell seeding. We observed a maximal number of non-adherent cells on ODS (Fig. 3a) and alcohol-treated surfaces (Fig. 3b), while RFGDT surfaces had the least numbers immediately following seeding. Time course analysis with the adhesion strength assays further validated this observation with silane (Fig. 3c) and alcohol-treated surfaces (Fig. 3d), demonstrating most nonadherent cells, while RFGDT offered the optimal biomaterial interface for cell adhesion (Fig. 3e). The pronounced increase in non-adherent cells in the alcohol-treated group at 5 hours could be attributed to $r$ persistent residual agents reducing cell viability. To validate the role of redox signaling, incubation with either N-Acetyl Cysteine or Catalase was noted to reduce RFGDT improved cell attachment (Fig. 3f).

\section{Improved cell adhesion is mediated via RFGDT-generated redox-mediated RPSA expression}


Cell adhesion involves concerted actions of multiple cell membrane and cytoplasmic molecules capable

of dynamic assembly of an attachment complex. ${ }^{28-30}$ Among these various mediators, laminin receptors on the cell surface have been shown to play a critical role in mediating matrix interactions. ${ }^{\left[{ }^{[7]}\right.}$ Elucidation of these adhesive complexes has also outlined several upstream signaling mediators that outline the role of a laminin receptor, Ribosomal Protein SA (RPSA), induced by redox signaling. ${ }^{31,32}$ Hence, to examine the effects of redox signaling on cell adhesion, MDPC-23 cells were treated with various concentrations of hydrogen peroxide that demonstrated increased RPSA and phospho-FAK 397 expression (Fig. 4a). Next, we examined if the RFGDT-improved cell adhesion could be mediated via a similar mechanism. We observed a robust increase in phospho-FAK ${ }_{397}$ and RPSA expression following RFGDT by immunoblotting (Fig. 4b) and immunofluorescence (Figs. 4c and 4d). These responses were abrogated by pretreatment of the biomaterial surface with silane or by the addition of NAC, indicating these are redox-mediated. Finally, we inquired if the improved cell attachment via the enhanced laminin receptor following RFGDT would affect cell survival and proliferation. We observed a significant improvement $(\mathrm{n}=$ $3, p<0.05)$ in RGFDT group compared to all other groups (Fig. 4e). These results suggest that improved cell adhesion following RFGDT could be mediated via induced RPSA expression.

\section{Discussion}

Biomaterial interfaces represent a major frontier for improving functional integration and determining clinical therapeutic outcomes. ${ }^{33}$ Significant progress in biomaterial composition and design has evolved from simple biocompatible, inert interfaces to existing bioactive surfaces. More recent biomaterial systems are focusing on smart systems that are capable of sensing-and-responding to biological scenarios with directed responses. A major functional characteristic of these biomaterial interfaces is to selectively promote desirable biological responses while actively dissuading detrimental ones. ${ }^{3}$ Some of the latter responses are directed at reducing microbial (biofilm) burden to prevent protracted inflammation or host immune responses. ${ }^{34,35}$ This not only facilitates routine tissue healing responses, but may enable regenerative clinical outcomes.

This study utilized common oral microbes and cells from the dental pulp to examine the effects of RFGDT on biomaterials used routinely in clinical dentistry. Pulpal inflammation due to caries results in irreversible necrosis, necessitating Endodontic intervention and coronal restorations. Various pulp capping agents such as calcium hydroxide, mineral trioxide aggregate (MTA), Emdogain, or glass ionomer cement provide cues to promote tissue healing (osteodentin). However, they do not directly address residual infection that leads to protracted inflammation. The prominent dual-action of RFGDT noted in this study namely antimicrobial disinfection and promoting cell adhesion and expansion is particularly attractive for clinical use (Fig 5). ${ }^{36,37}$ The antimicrobial actions of RFGDT predominantly targeting the catalase-negative microbes appear to be directly relevant to healthy clinical scenarios where aerobic bacteria predominate. The reduced efficacy on catalase-negative microbes was evident in this study could be clinically addressed with additional rinses or medications prior to material placement. The sudy noted the generation of redox species from biomaterial surfaces was based on their chemical 
composition. While both glass and platinum could induce redox, the polymeric (PMMA) substrate demonstrated minimal induction. This appears to reflect the ionization potential of the predominant elemental (Glass: Silica $786 \mathrm{Kj} / \mathrm{mol}$ and Platinum $867 \mathrm{Kj} / \mathrm{mol}$ ) constituents that are representative of dental biomaterials such as reinforced cement and metal implants. However, unlike these two biomaterials that intimately interact with cells, the polymeric material (PMMA: $6857 \mathrm{Kj} / \mathrm{mol}$ ) is predominantly used to fabricate external prosthetic devices where the disinfection and non-adhesive characteristics are of more significance. Nonetheless, direct composite materials as pulp dressings would appear to be unsuitable for the RFGDT technique.

We observed an optimal treatment time of three minutes for disinfection and improved cell adhesion with RFGDT for glass surfaces under vacuum. Besides the biomaterial composition and treatment time, other variables determining these interfacial responses include gaseous composition (such as helium or argon) within the chamber, energy density, and the presence of other trace agents. ${ }^{38,39}$ Attention to these parameters can enable development of future biomaterial-specific RFGDT protocols. It is also important to emphasize that the RFGDT was performed on inanimate biomaterial surfaces, before cell seeding, without direct exposure to microbes or cells. Direct treatments with non-thermal plasma on mammalian cells result in DNA damage and loss of viability. ${ }^{40}$ This essentially implies direct clinical RFGDT on human tissues for disinfection or improving cellular responses is an untenable strategy. Nonetheless, surface treatments of biomaterial devices could prime them for optimal tissue interactions such as osseointegration of metallic implants, glass ionomer, or metal-reinforced composites as dental filling materials. The latter application was a primary motivation in our choice of the pre-odontoblast cell line, MDPC-23. Interestingly, we have observed differential redox sensitivity among cells of discrete lineage in other studies. ${ }^{41}$ Future studies could examine the selectivity of RFGDT on complex multipartite biomaterial systems with specific cell types such as osteoblasts (osseointegration), keratinocytes (wound healing), fibroblasts (matrix production), or endothelial cells (angiogenesis) for directed clinical outcomes.

This study observed RFGDT-generated ROS detected using chemiluminescence following oxidation of luminol. ${ }^{28}$ The amounts and discrete ROS have critical roles in cell survival, migration, and apoptosis. ${ }^{42,43}$ Redox is a physiological regulator of intracellular signaling cascades, especially tyrosine kinase-mediated growth factors, has gained recent attention due to its ability to reversibly oxidize redox-sensitive targets. ${ }^{44}$ ] These reversible redox modifications of cysteine-residues in proteins, also termed thiol switches, are regulated by oxidoreductase and thiol-isomerases. ${ }^{45}$ Their primary function involves neutralizing excessive redox in the biological system that can lead to deleterious damage. Other targets of redoxgenerated signaling include a broad array of cytoskeletal components, signaling intermediates, and transcriptional regulators.

A major finding in this study is that RFGDT induced redox promotes laminin receptor, RPSA expression that appears to mediate the improved cell adhesion and survival. Laminins are high molecular weight extracellular glycoproteins that constitute the major non-collagenous component of basal lamina in 
cells. ${ }^{46,47}$ Their multidomain proteins are encoded by eleven human genes characterized by heterodimeric a, $\beta$ and $g$ chains that constitute 16 different isoforms. Their key contributions to various cellular functions such as adhesion, migration, and differentiation are well described. There have been recent insights into the roles of specific Laminins to selectively drive cellular enrichment and lineage specification that has potent implications for tissue healing and regeneration. ${ }^{48}$ Besides the prototypical members, there are a large number of proteins with laminin-like domains that include Ribosomal Protein SA (RPSA) that has also been known as LamR, 37LRP, and LBP/p40, among others. ${ }^{49}$ RPSA is a ribosomal protein with several pathophysiological functions in protein translation, cell adhesion, tumor cell invasion, viral cell entry, and small molecule sensing. ${ }^{50,51}$ The amino acid sequence is evolutionarily conserved with over $98 \%$ homology in all mammals and structural similarities with the prokaryotic gene, RPS2. ${ }^{49}$ It would be interesting to examine the functional significance of RFGDT induced RPS2 in the microbial species in future studies.

RPSA has been noted to be involved in cap-dependent eukaryotic translation, especially in transformed and viral infected cells. However, upstream induction of RPSA signaling remains to be fully elucidated. A few prior reports have noted RPSA is induced by redox-generated signaling, suggesting it us a redoxinduced target gene. ${ }^{31,32}$ Consistent with these reports, this study outliens the ability of RFGDT induced redox was able to increase RPSA expression. The increased expression of this cell adhesion receptor would contribute to improved attachment and survival of the MDPC-23 cells. Membrane clustering of these laminin receptors has been noted to induce tyrosine 397 phosphorylation of FAK, as was also noted in this study. Both FAK and Src activation at these membrane adhesion complexes further drive several cellular functions such as cell spreading, migration, proliferation, and prevention of apoptosis. Thus, the use of RFGDT appears to evoke discrete redox-generated biological responses that can be harnessed for both disinfection and cellular functions such as cell attachment, expansion, and differentiation to aid tissue regeneration.

In conclusion, this study demonstrates the ability of a non-invasive, directed energy tool, RFGDT, is capable of concurrent antimicrobial disinfection and improved directed cellular responses via redoxsignaling. This showcases RFGDT as a simple, cost-effective, and convenient bench-top tool for clinical application to improve tissue healing and regenerative outcomes.

\section{Materials \& Methods}

Cell Culture: Mouse dental pulp cells (MDPC-23) were maintained in DMEM (Glutamax, high-glucose) supplemented with $10 \%$ Fetal Bovine Serum (certified grade) and 100 units ml$^{-1}$ Penicillin and $100 \mu \mathrm{g} \mathrm{ml}^{-1}$ Streptomycin (all from Thermo-Scientific, USA). Cells were grown at $37^{\circ} \mathrm{C}$ in a humidified chamber with $5 \% \mathrm{CO}_{2}$.

Sample preparation and sterilization: Cover glass (18 mm, 0.12-0.17 mm thickness, Matsunami micro cover glass) were first cleaned with 10\% detergent (Sparkleen, Fisher Scientific, USA) and sonicated 
(Aristocrat Ultrasonic, Healthco, USA) for 10 minutes, followed by three washes in distilled water and allowed to dry vertically in a holder. Following this, some covers slips were soaked in $95 \%$ Ethanol (Sigma-Aldrich, USA) for 10 minutes, while another set was soaked in 2\% n-Octadecyl Trichlorosilane (ODS) for 10 minutes. Finally, another set of coverslips were subjected to RFGDT, as described below. Except for the RFGDT, all samples were sterilized under UV light or 15 minutes before cell seeding.

Radiofrequency Glow Discharge Treatments (RFGDT): A partial air-vacuum RFGD device (PDC-32G, Harrick Scientific, USA) was used as per the manufacturer's instructions. The biomaterials (glass coverslips, platinum sheets, or PMMA discs) were placed within the chamber and treated various times as indicated in individual studies. Biomaterial specimens were used immediately for biochemical, microbiological, or cell adhesion/survival studies.

AlamarBlue assay: MDPC-23 cells were seeded at a cell density of $1 \times 10^{5}$ cells $\mathrm{ml}^{-1}$ on the treated glass surfaces described above placed in a 12 well dish. Cell viability and proliferation were assessed with the AlamarBlue assay as per the manufacturer's instructions. Briefly, following 24 hours of cell incubation, $10 \%(\mathrm{v} / \mathrm{v}$ ) of AlamarBlue reagent (Thermo Fisher Scientific, USA) was added to complete media and incubated for 2 hours. Replicates of conditioned media from each well were transferred to black wells, and fluorescence was assessed using a plate reader (Victor3, Perkin Elmer, USA).

Cell Adhesion Assay: MDPC-23 cells were seeded at a cell density of $1 \times 10^{5}$ cells mll$^{-1}$ on the treated glass surfaces described above placed in a 12 well dish. Cell adhesion was assessed after 5 hours of incubation as described previously. Briefly, media was collected and assessed for floating (non-seeded) cells. After replacing the media, plates were subjected to centrifugation (Eppendorf, USA) at $2400 \mathrm{rpm}$ (weak adhesions) and $4000 \mathrm{rpm}$ (strong adhesions) for 6 minutes, and the media was collected to assess cell numbers. Images were also captured of these wells before and after spinning to document the number and morphology of the adherent cell populations. In some studies, Catalase (10 uM), N-Acetyl Cysteine (1 mM), or Hydrogen peroxide (all from Sigma-Aldrich, USA) were included in the media prior to cell seeding of the glass surfaces to assess the role of RFGDT-induced ROS in mediating cell adhesion.

ROS Detection assay: To assess RFGDT-generated ROS, a chemiluminescence assay with Luminol (detects both extra- and intracellular ROS) was utilized as described previously. ${ }^{[33]}$ Briefly, a solution was prepared by mixing $5 \mathrm{mg}$ of luminol powder with $100 \mathrm{~mL}$ of $1 \mathrm{M}$ Sodium hydroxide (both Sigma-Aldrich, USA) and mixed gently. The freshly prepared luminol solution was added to the cells in a 12 wells plate at a ratio of $1 \mathrm{~mL} /$ well $(\mathrm{v} / \mathrm{v})$ immediately after RFGDT treatments at different doses, and chemiluminescence was detected at $0.5,1,3$, and 5 minutes using a multiwell detector (Victor3, Perkin Elmer, USA).

Western blotting: Glass coverslips were RFGDT treated and seeded with MDPC23 cells. After 5 hours of incubation in $\mathrm{CO}_{2}$ incubator, the attached cells on the coverslip were collected from different groups, and collected cells were re-suspended in RIPA buffer (Sigma-Aldrich, USA) with Mini Protease Inhibitor (Thermo Scientific, USA). Cells were disturbed with two cycles of sonication (QSonica, USA) for 5 seconds each, and lysates were centrifuged at $14,000 \mathrm{rpm}$ at four ${ }^{\circ} \mathrm{C}$ for 20 minutes. The total protein in the 
lysates was quantified with the Bradford assay kit (BCA Protein Assay, Thermo Scientific Inc). Protein lysates were separated in precast mini-protein TGX stain-free gels and transferred to PVDF membranes (both Bio-Rad, USA). Blots were initially blocked with 1\% BSA for 1 hour and further incubated with primary antibodies for Ribosomal Protein SA (RPSA, Abcam, USA) or phospho-FAK (Cell signaling, USA) at $4^{\circ} \mathrm{C}$ overnight. Following washes, blots were incubated for normalization with appropriate speciesspecific HRP conjugated secondary antibodies (Cell signaling, USA) or HRP-conjugated $\beta$-actin (Cell signaling, USA). Finally, the blots were developed by using chemiluminescent substrates (Thermo Scientific, USA), and images were scanned digitally using ChemiDoc MP Imaging System (Bio-Rad Laboratories, USA).

Immunofluorescence staining: For immunostaining, the RPSA antibody (Abcam, USA) was incubated for 1 hour, followed by Alexa Fluor 488 secondary antibody (Cell signaling, USA), counterstained with DAPI, and images were captured using Fluorescence Cell Imager (ZOE, Bio-Rad, USA).

Microbial viability assay: Facultative anaerobes such as Streptococcus mutants (S. mutants), Streptococcus gordonii DL1 (S. Gordonni), Moraxella catarrhalis (M. catarrhalis) were grown in brain heart infusion medium (BD Bioscience, USA) at $37^{\circ} \mathrm{C}$ and Obligate anaerobe Porphyromonas gingivalis ( $P$. gingivalis) was grown anaerobically in trypticase soy broth medium (BD Bioscience, USA) supplemented with $10 \%$ sheep blood, hemin $\left(5 \mathrm{\mu g} \mathrm{ml}^{-1}\right)$ and menadione $\left(1 \mu \mathrm{g} \mathrm{ml}^{-1}\right)$ and incubated at $37^{\circ} \mathrm{C}$ in an atmosphere of $\mathrm{N}_{2} / \mathrm{H}_{2} / \mathrm{CO}_{2}$ (90:5:5). A single colony of each bacterium was added to $5 \%$ or $15 \%$ of $\mathrm{H}_{2} \mathrm{O}_{2}$ and qualitatively analyzed for the presence of antioxidant catalase. For the survival assay, a 10\% 2,3,5Triphenyl-tetrazolium chloride solution (TTC, Sigma Aldrich, USA) was added to cultures wells and incubated for 6-12 hours or until red color change was apparent. Color intensity was quantified by using a microplate reader (SpectraMax i3x, Molecular device, USA) at 480nm.

Microbial survival assay: For the CFU assays, coverslips were inoculated with $250 \mathrm{uL}$ respective bacterial culture and incubated in species-specific growth conditions for 24 hours (S. gordonni, M. catarrhalis, $S$. mutants) or 5 days (P. gingivalis). Aliquots of bacterial culture from glass coverslips were serially diluted by 10 -fold dilutions and plated via the drop plate technique.

Statistical Analyses: Data were analyzed using Excel (Microsoft, USA), and statistical significance was assessed using either a one-way ANOVA or T-Tests, where $p<0.05$ was considered statistically significant. All studies were repeated at least twice, with each assay performed in minimum duplicates.

\section{Declarations}

Acknowledgements: This work was supported by a faculty start-up grant (PRA).

Conflicts of Interest: The authors declare they have no conflicts of interest.

Avaialbility of data and materials: All data generated or analysed during this study are included in this published article and its supplementary information files. Any further information or material are however 
available from the authors upon reasonable request.

\section{References}

1. Discher, D. E., Mooney, D. J. \& Zandstra, P. W. Growth factors, matrices, and forces combine and control stem cells. Science 324, 1673-1677, doi:10.1126/science.1171643 (2009).

2. Langer, R. \& Vacanti, J. P. Tissue engineering. Science 260, 920-926 (1993).

3. Arany, P. R. \& Mooney, D. J. At the edge of translation - materials to program cells for directed differentiation. Oral Dis 17, 241-251, doi:10.1111/j.1601-0825.2010.01735.x (2011).

4. Rahman, S. U., Nagrath, M., Ponnusamy, S. \& Arany, P. R. Nanoscale and Macroscale Scaffolds with Controlled-Release Polymeric Systems for Dental Craniomaxillofacial Tissue Engineering. Materials (Basel) 11, doi:10.3390/ma11081478 (2018).

5. Lutolf, M. P. \& Hubbell, J. A. Synthetic biomaterials as instructive extracellular microenvironments for morphogenesis in tissue engineering. Nat Biotechnol 23, 47-55, doi:10.1038/nbt1055 (2005).

6. Srivastava, D. \& DeWitt, N. In Vivo Cellular Reprogramming: The Next Generation. Cell 166, 13861396, doi:10.1016/j.cell.2016.08.055 (2016).

7. Stevens, M. M. et al. In vivo engineering of organs: the bone bioreactor. Proc Natl Acad Sci U S A 102, 11450-11455, doi:10.1073/pnas.0504705102 (2005).

8. Takahashi, K. \& Yamanaka, S. Induction of pluripotent stem cells from mouse embryonic and adult fibroblast cultures by defined factors. Cell 126, 663-676, doi:10.1016/j.cell.2006.07.024 (2006).

9. Garrett, W. S. et al. Communicable ulcerative colitis induced by T-bet deficiency in the innate immune system. Cell 131, 33-45, doi:10.1016/j.cell.2007.08.017 (2007).

10. Macpherson, A. J. et al. A primitive T cell-independent mechanism of intestinal mucosal IgA responses to commensal bacteria. Science 288, 2222-2226, doi:10.1126/science.288.5474.2222 (2000).

11. Mazmanian, S. K., Liu, C. H., Tzianabos, A. O. \& Kasper, D. L. An immunomodulatory molecule of symbiotic bacteria directs maturation of the host immune system. Cell 122, 107-118, doi:10.1016/j.cell.2005.05.007 (2005).

12. Reinhardt, C., Reigstad, C. S. \& Backhed, F. Intestinal microbiota during infancy and its implications for obesity. J Pediatr Gastroenterol Nutr 48, 249-256, doi:10.1097/mpg.0b013e318183187c (2009).

13. Sudo, N. et al. The requirement of intestinal bacterial flora for the development of an IgE production system fully susceptible to oral tolerance induction. J Immunol 159, 1739-1745 (1997).

14. Dewhirst, F. E. et al. The human oral microbiome. J Bacteriol 192, 5002-5017, doi:10.1128/JB.00542-10 (2010).

15. Genco, R. J., Grossi, S. G., Ho, A., Nishimura, F. \& Murayama, Y. A proposed model linking inflammation to obesity, diabetes, and periodontal infections. J Periodonto/ 76, 2075-2084, doi:10.1902/jop.2005.76.11-S.2075 (2005). 
16. Jenkinson, H. F. \& Lamont, R. J. Oral microbial communities in sickness and in health. Trends Microbiol 13, 589-595, doi:10.1016/j.tim.2005.09.006 (2005).

17. Latimer, J. M. et al. Regenerative Medicine Technologies to Treat Dental, Oral, and Craniofacial Defects. Front Bioeng Biotechnol 9, 704048, doi:10.3389/fbioe.2021.704048 (2021).

18. Martins, N. \& Rodrigues, C. F. Biomaterial-Related Infections. J Clin Med 9, doi:10.3390/jcm9030722 (2020).

19. Rimondini, L., Fini, M. \& Giardino, R. The microbial infection of biomaterials: A challenge for clinicians and researchers. A short review. J Appl Biomater Biomech 3, 1-10 (2005).

20. Dai, Z., Ronholm, J., Tian, Y., Sethi, B. \& Cao, X. Sterilization techniques for biodegradable scaffolds in tissue engineering applications. J Tissue Eng 7, 2041731416648810, doi:10.1177/2041731416648810 (2016).

21. Tao, M. et al. Sterilization and disinfection methods for decellularized matrix materials: Review, consideration and proposal. Bioact Mater 6, 2927-2945, doi:10.1016/j.bioactmat.2021.02.010 (2021).

22. Langmuir, I. Oscillations in Ionized Gases. Proc Natl Acad Sci U S A 14, 627-637, doi:10.1073/pnas.14.8.627 (1928).

23. Mott-Smith, H. M. History of "plasmas". Nature 233, 219, doi:10.1038/233219a0 (1971).

24. Lai, H. C. et al. The influence of surface energy on early adherent events of osteoblast on titanium substrates. J Biomed Mater Res A 93, 289-296, doi:10.1002/jbm.a.32542 (2010).

25. Ali, H., Baier, R. E., Dziak, R. \& Andreana, S. Promotion of cells to close gaps and encourage cell coverage, by radio frequency glow discharge treatment. J Biomed Mater Res A 105, 169-177, doi:10.1002/jbm.a.35888 (2017).

26. Baier, R. E. \& Meyer, A. E. Implant surface preparation. Int J Oral Maxillofac Implants 3, 9-20 (1988).

27. Baier, R. E., Meyer, A. E., Natiella, J. R., Natiella, R. R. \& Carter, J. M. Surface properties determine bioadhesive outcomes: methods and results. J Biomed Mater Res 18, 337-355, doi:10.1002/jbm.820180404 (1984).

28. Bedouhene, S., Moulti-Mati, F., Hurtado-Nedelec, M., Dang, P. M. \& El-Benna, J. Luminol-amplified chemiluminescence detects mainly superoxide anion produced by human neutrophils. Am J Blood Res 7, 41-48 (2017).

29. Gumbiner, B. M. Cell adhesion: the molecular basis of tissue architecture and morphogenesis. Cell 84, 345-357, doi:10.1016/s0092-8674(00)81279-9 (1996).

30. Zaidel-Bar, R. Cadherin adhesome at a glance. J Cell Sci 126, 373-378, doi:10.1242/jcs.111559 (2013).

31. Vilas-Boas, F. et al. Hydrogen peroxide regulates cell adhesion through the redox sensor RPSA. Free Radic Biol Med 90, 145-157, doi:10.1016/j.freeradbiomed.2015.11.019 (2016).

32. Kang, H. S. et al. LRP1-dependent pepsin clearance induced by 2'-hydroxycinnamaldehyde attenuates breast cancer cell invasion. Int J Biochem Cell Biol 53, 15-23, 
doi:10.1016/j.biocel.2014.04.021 (2014).

33. Tibbitt, M. W., Rodell, C. B., Burdick, J. A. \& Anseth, K. S. Progress in material design for biomedical applications. Proc Natl Acad Sci U S A 112, 14444-14451, doi:10.1073/pnas.1516247112 (2015).

34. Li, W., Thian, E. S., Wang, M., Wang, Z. \& Ren, L. Surface Design for Antibacterial Materials: From Fundamentals to Advanced Strategies. Adv Sci (Weinh), e2100368, doi:10.1002/advs.202100368 (2021).

35. Franz, S., Rammelt, S., Scharnweber, D. \& Simon, J. C. Immune responses to implants - a review of the implications for the design of immunomodulatory biomaterials. Biomaterials 32, 6692-6709, doi:10.1016/j.biomaterials.2011.05.078 (2011).

36. Baier, R. E. Principles of adhesion. Oper Dent Suppl 5, 1-9 (1992).

37. Baier, R. E. Surface behaviour of biomaterials: the theta surface for biocompatibility. J Mater Sci Mater Med 17, 1057-1062, doi:10.1007/s10856-006-0444-8 (2006).

38. Shibata, Y., Hosaka, M., Kawai, H. \& Miyazaki, T. Glow discharge plasma treatment of titanium plates enhances adhesion of osteoblast-like cells to the plates through the integrin-mediated mechanism. Int J Oral Maxillofac Implants 17, 771-777 (2002).

39. Yamamoto, H., Shibata, Y. \& Miyazaki, T. Anode glow discharge plasma treatment of titanium plates facilitates adsorption of extracellular matrix proteins to the plates. J Dent Res 84, 668-671, doi:10.1177/154405910508400717 (2005).

40. Kalghatgi, S. et al. Effects of non-thermal plasma on mammalian cells. PLoS One 6, e16270, doi:10.1371/journal.pone.0016270 (2011).

41. Engel, K. W., Khan, I. \& Arany, P. R. Cell lineage responses to photobiomodulation therapy. J Biophotonics 9, 1148-1156, doi:10.1002/jbio.201600025 (2016).

42. Chiu, J. \& Dawes, I. W. Redox control of cell proliferation. Trends Cell Biol 22, 592-601, doi:10.1016/j.tcb.2012.08.002 (2012).

43. Trachootham, D., Lu, W., Ogasawara, M. A., Nilsa, R. D. \& Huang, P. Redox regulation of cell survival. Antioxid Redox Signal 10, 1343-1374, doi:10.1089/ars.2007.1957 (2008).

44. Chiarugi, P. Reactive oxygen species as mediators of cell adhesion. Ital J Biochem 52, 28-32 (2003).

45. Lorenzen, I., Eble, J. A. \& Hanschmann, E. M. Thiol switches in membrane proteins - Extracellular redox regulation in cell biology. Biol Chem 402, 253-269, doi:10.1515/hsz-2020-0266 (2021).

46. Aumailley, M. The laminin family. Cell Adh Migr 7, 48-55, doi:10.4161/cam.22826 (2013).

47. Timpl, R. et al. Laminin--a glycoprotein from basement membranes. J Biol Chem 254, 9933-9937 (1979).

48. Ohta, R. et al. Laminin-guided highly efficient endothelial commitment from human pluripotent stem cells. Sci Rep 6, 35680, doi:10.1038/srep35680 (2016).

49. Satoh, K. et al. Cloning of 67-kDa laminin receptor cDNA and gene expression in normal and malignant cell lines of the human lung. Cancer Lett 62, 199-203, doi:10.1016/0304-3835(92)90096e (1992). 
50. Legler, D. [Physical therapy in children with asthma and mucoviscidosis]. Kinderkrankenschwester 11, 70-71 (1992).

51. Tachibana, H., Koga, K., Fujimura, Y. \& Yamada, K. A receptor for green tea polyphenol EGCG. Nat Struct Mol Biol 11, 380-381, doi:10.1038/nsmb743 (2004).

\section{Figures}

\section{Figure 1}

\section{RFGDT generates redox from biomaterial surfaces}

ROS generation was assessed with Luminol chemiluminescent assay immediately after RFGDT with various biomaterials namely (a) glass, (b) metal and (c) polymer; (d) ROS generation was also assessed following varying treatment times with RFGDT; (e) Glass surfaces pretreated with ODS, alcohol, or RFGDT were assessed with Luminol assay.

\section{Figure 2}

\section{Antimicrobial effects of RFGDT}

(a) Four bacterial species were subjected to hydrogen peroxide, and effervescence was assessed; (b) bacterial viability test with TTC assay was performed with $S$. mutans following RFGDT. Colony-forming assays were performed with four bacterial species following RFGDT namely (c) Streptococcus mutants, (d) Streptococcus gordonni, (e) Moraxella catarrhalis (f) Porphyromonas gingivalis. In some cases, incubation with N-Acetyl cysteine or catalase was performed, followed by RFGDT. $n=3,{ }^{*} p<0.05$. RFGDT: Radiofrequency glow discharge treatment; NAC: N Acetylcysteine; CFUs: Colony-forming units.

\section{Figure 3}

\section{Cell adhesion following RFGDT}

(a) MDPC-23 cells were seeded on these surfaces and assessed with microscopy; (b) Cell adherant to various treatmed biomaterial surfaces were assessed by cell counting; (c) Cell-seeded surfaces were subjected to low $(2400 \mathrm{rpm}$ ) centrifugal forces to determine weak cell adhesions at 5 hours; (d) Cellseeded surfaces were subjected to high $(4000 \mathrm{rpm})$ centrifugal forces to determine strong cell adhesions at 5 hours and (e) 24 hours. f) Strong cell adhesion was assessed with glass surfaces pretreated with NAC, Catalase, or hydrogen peroxide before RFGDT. All studies, $n=3$, * $p<0.05$. RFGD: Radiofrequency 
glow discharge; ODS: n-Octadecyl Trichlorosilanel; RFUs: Relative fluorescence units; NAC: N Acetylcysteine.

\section{Figure 4}

\section{Redox generated by RFGDT induces RPSA expression}

(a) Western blots for RPSA and activated FAK expression was assessed following hydrogen peroxide treatments over time; (b) Similar analysis was performed following RFGDT on glass surfaces. Some samples were pretreated with ODS or NAC before RFGDT and cell seeding; (c) Immunofluorescence for RPSA expression was performed on these glass surfaces following pretreatments or RFGDT; (d) quantification in five high power fields as means with standard deviations; (e) Glass surfaces were subjected to silane, alcohol, or RFGDT and seeded with MDPC-23 cells. Cell numbers were assessed with AlamarBlue assay after 24 hours. $n=3, * p<0.05$. RFGDT: Radiofrequency glow discharge treatment; ODS: n-Octadecyl Trichlorosilanel; NAC: N Acetylcysteine; DAPI: 4',6-diamidino-2-phenylindole.

\section{Figure 5}

\section{Outline of RFGDT responses}

The image shows that redox generated by RFGDT has a dual action with an antimicrobial effect on oral bacteria while inducing the laminin adhesion receptor, RPSA facilitating improved cell attachment and expansion of odontoblasts.

\section{Supplementary Files}

This is a list of supplementary files associated with this preprint. Click to download.

- Ponnusamyetal01192022Supporting.pdf 